\title{
Micromagnetics on curved geometries using rectangular cells: error correction and analysis
}

\author{
M. J. Donahue and R. D. McMichael \\ National Institute of Standards and Technology, Gaithersburg, MD 20899-8910
}

\begin{abstract}
This paper presents an edge field correction for micromagnetic computations of arbitrarily shaped objects on rectangular grids. The correction is compatible with FFT techniques and involves factors that are precomputed using the standard self-magnetostatic algorithms applied on a local, refined mesh. To evaluate this correction, we introduce a quantitative measure that is based on calculating an edge mode resonance for different orientations of an edge with respect to the rectangular mesh. Applied to a $350 \mathrm{~nm} \mathrm{Ni} 80 \mathrm{Fe}_{20}$ square, we find up to a $50 \%$ frequency shift for the uncorrected approach, but less than a 5\% shift using the proposed method. We also study vortex expulsion in a $220 \mathrm{~nm} \mathrm{Ni} \mathbf{N i}_{80} \mathrm{Fe}_{20}$ square, and again find that the proposed correction significantly reduces the dependence of the expulsion field on the orientation angle of the sample square with the mesh.
\end{abstract}

Index Terms-Micromagnetic modeling, edge corrections, magnetic edge properties

\section{INTRODUCTION}

Regular grids are attractive for micromagnetic modeling because they enable fast and efficient computation of the long range magnetostatic interactions using fast Fourier transform (FFT) techniques[1]. However, regular grids carry a disadvantage that only shapes that coincide with the grid boundaries are nicely modeled. For shapes that do not fit neatly on the grid, a simple approximation to the part shape can be made by setting saturation magnetization $M_{\mathrm{s}}=0$ in all cells with centers outside the desired part boundary, while maintaining the full value of $M_{\mathrm{s}}$ inside the boundary. The approximated part shape will then have a stair-step profile characteristic of the discretization grid as shown in Fig. 1.

Unfortunately, the stray field computed using the stair-step approximation differs significantly from the field produced

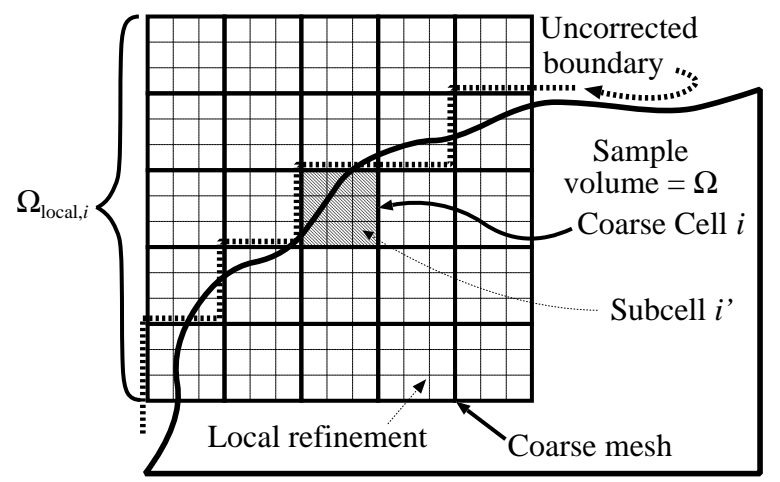

Fig. 1. A refined mesh is used to calculate the magnetostatic fields in the neighborhood $\Omega_{\text {local }, i}$ of partially filled edge cells. by an ideal edge. This problem is largely avoided by finite element techniques, which allow a much better approximation of arbitrary shapes, at the expense of a less efficient calculation of magnetostatic fields[2], [3]. An alternative is to provide corrections within a finite difference code to compensate for the effects of stair-step edge contours[4], [5]. In this paper we introduce and evaluate a new correction method of the latter type that allows a finite difference model to more closely approximate the micromagnetics of ideal edges.

\section{STAIR-STEP CORRECTION METHOD}

The method presented here involves separating the magnetostatic field calculation into a rough, long range part and a refined short range part. The separation is made in a manner that allows the long range part to be computed by efficient FFT methods, while the near field correction term ensures accurate values in the short range. The short range correction involves factors computed during problem initialization that are based on pointwise local submeshes that are many times finer than the base mesh, as schematically illustrated in Fig. 1. However, at run time both short and long range parts are computed on a mesh that is sized at the usual micromagnetic length scale, i.e., on the order of the material exchange length.

We begin by considering the usual demagnetization energy that would be computed on a fine mesh that spans the entire problem domain:

$$
E_{\text {demag }}=\frac{1}{2} \mu_{0} V_{\text {fine }} \sum_{i^{\prime}} \sum_{j^{\prime}} \mathbf{M}_{i^{\prime}}^{T} N_{i^{\prime} j^{\prime}} \mathbf{M}_{j^{\prime}}
$$

where $V_{\text {fine }}$ is the volume of the submesh cells, $N_{i^{\prime} j^{\prime}}$ is the demagnetization tensor[6] interaction between subcells $i^{\prime}$ and $j^{\prime}$, and $\mathbf{M}_{i^{\prime}}$ is the magnetization in subcell $i^{\prime}$. If we define the constant $C_{F}$ to be the number of subcells contained in one coarse mesh cell, or, equivalently, $C_{F}=V_{\text {coarse }} / V_{\text {fine }}$, then we can define a corresponding averaged interaction tensor $L_{i j}$ between coarse cells $i$ and $j$ by

$$
L_{i j}=\frac{C_{F}}{\left(\sum_{i^{\prime} \in i} \eta_{i^{\prime}}\right)\left(\sum_{j^{\prime} \in j} \eta_{j^{\prime}}\right)} \sum_{i^{\prime} \in i} \sum_{j^{\prime} \in j} \eta_{i^{\prime}} \eta_{j^{\prime}} N_{i^{\prime} j^{\prime}} .
$$

Here we have defined a factor $\eta_{i}^{\prime}$ which takes on value 1 if subcell $i^{\prime}$ is inside the sample volume $\Omega$, or 0 otherwise.

Because the coarse mesh is assumed fine enough to capture the variation in the magnetization, we will assume that the reduced magnetization $\mathbf{m}_{i^{\prime}}$ is constant across the enclosing coarse mesh cell $i$. If we let $\mathbf{M}_{i}$ denote the average magnetization in cell $i$, i.e., $\mathbf{M}_{i}=\mathbf{m}_{i} v_{i} M_{s}$ where $v_{i}$ is the fraction 
of cell $i$ volume that is inside the sample boundary, then we can rewrite (1) as

$$
E_{\text {demag }}=\frac{1}{2} \mu_{0} V_{\text {coarse }} \sum_{i} \sum_{j} \mathbf{M}_{i}^{T} L_{i j} \mathbf{M}_{j} .
$$

This expression possesses the accuracy of the fine mesh, but is computed on the coarse mesh. Unfortunately, the interaction tensor $L_{i j}$ is not translation invariant ( $L_{i j}$ depends on $i$ and $j$ independently, as opposed to depending only on the difference $i-j$ ), so (3) cannot be efficiently computed using an FFT.

However, in the far field, where the distance between cells $i$ and $j$ is large relative to the cell size, the tensor elements $L_{i j}$ are well approximated by $N_{i j}$, where $N_{i j}$ is the usual demagnetization tensor for coarse mesh cells $i$ and $j$. Indeed, the net dipole moment from an averaged magnetization cell, $\mathbf{M}_{i}$, is the same as that from the corresponding fine mesh cell collection $\mathbf{M}_{i^{\prime}}$, so the leading term of the difference is a quadrupole field. Moreover, $N_{i j}$ is translation invariant, so the following scheme suggests itself. First, compute a rough demagnetization field using the coarse cell volume averaged magnetizations $\mathbf{M}_{i}$ and the standard coarse mesh demagnetization tensor:

$$
\mathbf{H}_{\text {demag,i }}^{\text {rough }}=-\sum_{j} N_{i j} \mathbf{M}_{j} \text {. }
$$

This can be computed efficiently using FFT techniques, and accurately represents the contribution from cells $j$ far removed from cell $i$. However, the contribution from nearby cells is inaccurate, so for cells near $i$, say $j \in \Omega_{\text {local }, i}$, we add in a correction term

$$
\mathbf{H}_{\mathrm{corr}, i}=\sum_{j \in \Omega_{\mathrm{local}, i}}\left(N_{i j}-L_{i j}\right) \mathbf{M}_{j} .
$$

The final computed value for the demagnetization field is then

$$
\mathbf{H}_{\text {demag,i }}=\mathbf{H}_{\text {demag }, i}^{\text {rough }}+\mathbf{H}_{\text {corr }, i} \text {. }
$$

This expression computes the near field contribution $(j \in$ $\left.\Omega_{\text {local }, i}\right)$ in exact agreement with that computed from the fine mesh. The difference in the far field contribution can be made arbitrarily small by enlarging $\Omega_{\text {local }, i}$ as desired.

Although $\mathbf{H}_{\text {corr, } i}$ cannot be computed using FFT techniques, it is not too expensive to compute because it involves only a local sum across $\Omega_{\text {local }, i}$. Moreover, $\mathbf{H}_{\text {corr }, i}$ only needs to be computed for those cells $i$ such that their local neighborhood $\Omega_{\text {local }, i}$ intersects the boundary, since if $\Omega_{\text {local }, i}$ is entirely contained inside the sample volume $\Omega$, then $\mathbf{H}_{\text {corr }, i}$ will be zero. It should also be noted that although (1) was derived using a fine mesh that covered the entire sample, to compute $\mathbf{H}_{\text {corr }}$ in practice only requires knowledge of the fine mesh $N_{i^{\prime} j^{\prime}}$ tensor elements across the much smaller local regions $\Omega_{i}$. Also, the $N_{i^{\prime} j^{\prime}}$ elements are the same for all $\Omega_{i}$; the variation in $\mathbf{H}_{\mathrm{corr}, i}$ for different $i$ come about due to different $\eta_{i^{\prime}} \eta_{j^{\prime}}$ in (2), not different $N_{i^{\prime} j^{\prime}}$.

The decomposition discussed here is similar to the method proposed in [5], except that in the present work the local field correction is computed using the same algorithm as is used for the long range part, as opposed to requiring development of a separate, special algorithm to handle curved edges. Also, the far field approximation in (4) includes local field contributions, so the correction term (5) only needs to be applied near the sample boundaries. Moreover, the current method can be used in $3 \mathrm{D}$ computations.

The cost in computing (5) can be further reduced by a simple approximation that works surprisingly well. First, break out the $\mathbf{m}_{i}$ dependent part:

$$
\begin{aligned}
\mathbf{H}_{\mathrm{corr}, i}= & \sum_{j \in \Omega_{\mathrm{local}, i}}\left(L_{i j}-N_{i j}\right) M_{s} v_{j}\left(\mathbf{m}_{i}-\mathbf{m}_{j}\right) \\
& -\sum_{j \in \Omega_{\mathrm{local}, i}}\left(L_{i j}-N_{i j}\right) M_{s} v_{j} \mathbf{m}_{i} .
\end{aligned}
$$

If the material is exchange coupled, then for cells $j$ close to cell $i$, the difference $\mathbf{m}_{i}-\mathbf{m}_{j}$ will be small, so the first term on the righthand side of (7) will be small. Even for cells on the periphery of $\Omega_{\text {local }, i}$, where $\mathbf{m}_{j}$ begins to vary more from $\mathbf{m}_{i}$, this term will be attenuated by the convergence of $L_{i j}$ towards $N_{i j}$. This shows that $\mathbf{H}_{\text {corr }, i}$ can be reasonably approximated by

$$
\mathbf{H}_{\mathrm{corr}, i} \approx-K_{i} \mathbf{M}_{i}
$$

where

$$
K_{i}:=\sum_{j \in \Omega_{\mathrm{local}, i}} \frac{v_{j}}{v_{i}}\left(L_{i j}-N_{i j}\right) .
$$

Thus $\mathbf{H}_{\mathrm{corr}, i}$ is reduced to the form of a simple cell-local anisotropy. This is essentially a generalization of the approximation used in [7] to model film thickness variation. This is the form for $\mathbf{H}_{\mathrm{corr}, i}$ that is used in the remainder of the paper. As will be seen in the next section, it works remarkably well. However, if higher accuracy is required, then (5) may be used directly, or else a higher order approximation to $\mathbf{m}_{j}$ may be introduced into (7).

\section{VALIDATION}

We use two methods to probe the properties of corrected and uncorrected edges in thin film squares. The first method measures the frequency of a spinwave mode localized near the film edge[8], [9], and the second method measures the field required to annihilate a vortex at the edge.

The edge mode frequency may be the less intuitive method, but it avoids numerical problems encountered near critical fields while providing a quantitative measure that is sensitive to the conditions on the edge. Further, the edge mode frequency is a natural response of the system, more closely related to the behavior of the magnetization than an arbitrary choice such as a field measurement at a chosen location.

Figures 2a)-c) illustrate a few of the normal modes of a square of $350 \mathrm{~nm}$ square of $\mathrm{Ni}_{80} \mathrm{Fe}_{20}\left(M_{\mathrm{s}}=800 \mathrm{kA} / \mathrm{m}, A\right.$ $=13 \mathrm{pJ} / \mathrm{m}$, damping parameter $\alpha=0.01), 5 \mathrm{~nm}$ thick in an applied field of $0.1 \mathrm{~T}$ and computed using $5 \mathrm{~nm}$ cubic cells. The spinwave mode spectrum and images are determined using a ground-pulse-ring-FFT sequence of computations that are described more fully elsewhere[10]. The greyscale in the mode images represents the out-of-plane component of the dynamic magnetization in the indicated modes.

Figure 2f) shows the lowest edge mode frequency as a function of the system rotation angle. At $\theta=0$, the part boundaries 

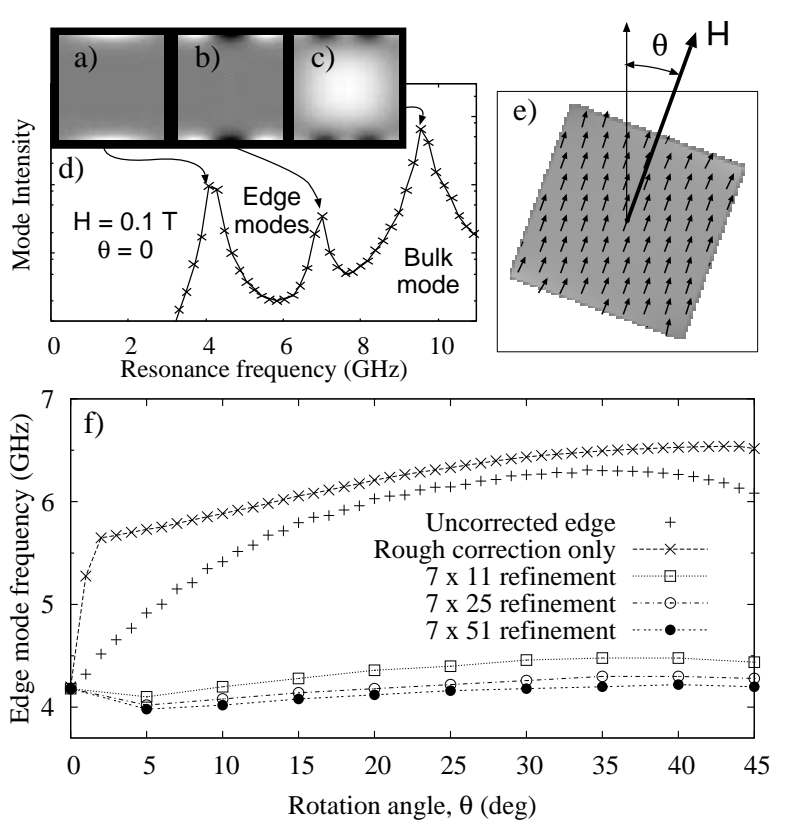

Fig. 2. Edge mode frequencies are used to test the edge corrections. A few of the lowest frequency spinwave modes for a $350 \mathrm{~nm}$ square of $\mathrm{Ni}_{80} \mathrm{Fe}_{20}, 5$ $\mathrm{nm}$ thick are shown in a)-c) with the mode spectrum in d) insets. The rotation angle $\theta$ is defined in e), and the frequency of the lowest frequency edge mode is plotted in f) for uncorrected stair-step edges, long-range corrections using diluted edge cells and the full correction using the locally refined grid. The $p \times q$ notation indicates a $p \times p$ local neighborhood with $q \times q$ subcells.

coincide perfectly with cell boundaries. For uncorrected edges and for the long range correction, the frequency increases by roughly $50 \%$ as the square and applied field are rotated out of alignment with the discretization grid.

The mechanism for the increase in precession frequency involves changes in the demagnetization field near the film edge. For the uncorrected edge and for the diluted edge cells, the demagnetization field near the edge is reduced as the magnetostatic charges are spread out either over the longer stair-step edge perimeter or spread over boundaries between cells with different magnetization. As the demagnetization field is reduced, the net field near the edge is increased, and therefore the precession occurs at a higher frequency. The edge mode frequency shift produced by edge property changes is expected to depend on a number of parameters including the applied field.

The corrected curves in Fig. 2f show the edge mode frequency when the short range correction is implemented as a local anisotropy term (8) for different different fine meshes. Local neighborhood size $p$ was found to be less important. The corrected edge mode frequencies vary by less than $5 \%$.

Within an excited edge mode, the spins remain nearly uniform, and it follows that the edge mode frequency test does not strongly test the assumption that is made in the derivation of (8) that the magnetization is nearly uniform near the edge.

To provide a stronger test of the assumption of nearly uniform magnetization near the edge, we calculated the field required to annihilate a vortex at the edge as a function of the system rotation angle. We modeled $220 \mathrm{~nm}$ squares of $2.5 \mathrm{~nm}$ thick $\mathrm{Ni}_{80} \mathrm{Fe}_{20}$ using $2.5 \mathrm{~nm}$ cubic cells with the

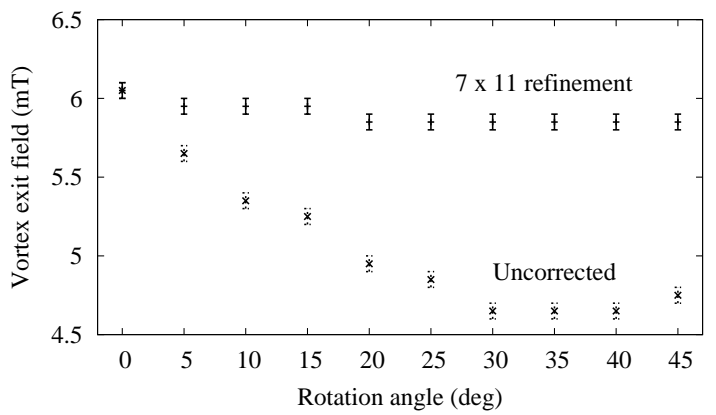

Fig. 3. The vortex exit field is used as a test of edge corrections. The field required to push a vortex out of a $220 \mathrm{~nm}$ square, $2.5 \mathrm{~nm}$ thick is plotted as a function of rotation angle. The error bars indicate the size of the field step.

applied field and square edges rotated relative to the grid as in the edge mode test. The smaller cells were needed to resolve the vortex core and minimize pinning of the vortex on the grid. Beginning with a vortex state, we calculated the vortex annihilation field as a function of the rotation angle. See Fig. 3. For the uncorrected edge, the vortex annihilation field drops by $26 \%$, while for the local edge correction, the change is only $3.2 \%$.

\section{CONCLUSION}

This paper presents a 3D-compatible correction method for calculating the micromagnetics of arbitrary shapes using regular finite-difference discretization. The correction reuses the magnetostatic code on a finer mesh to compute local anisotropy terms for cells in the neighborhood of the part boundary. The edge mode frequency test reveals dramatic gridinduced anisotropy that is largely suppressed by the correction.

This work supported in part by the NIST Magnetic Sensors Competence Program.

\section{REFERENCES}

[1] T. G. Stockham, "High speed convolution and correlation," Joint Computer Conference Proceedings, vol. 28, pp. 229-233, 1966.

[2] T. R. Koehler and D. R. Fredkin, "Finite element methods for micromagnetics," IEEE Trans. Mag., vol. 28, pp. 1239-1244, 1992.

[3] W. Scholz, J. Fidler, T. Schrefl, D. Suess, R. Dittrich, H. Forster, and V. Tsiantos, "Scalable parallel micromagnetic solvers for magnetic nanostructures," Comp. Mat. Sci, vol. 28, pp. 366-383, 2003.

[4] G. J. Parker, C. Cerjan, and D. W. Hewett, "Embedded curve boundary method for micromagnetic simulations," J. Magn. Magn. Mater, vol. 214, pp. 130-138, 2000.

[5] C. J. García-Cervera, Z. Gimbutas, and W. E, "Accurate numerical methods for micromagnetics simulations with general geometries," $J$. Comp. Phys., vol. 184, pp. 37-52, 2003.

[6] A. J. Newell, W. Williams, and D. J. Dunlop, "A generalization of the demagnetizing tensor for nonuniform magnetization," J. Geophys. Res.Solid Earth, vol. 98, pp. 9551-9555, 1993.

[7] D. G. Porter and M. J. Donahue, "Generalization of a two-dimensional micromagnetic model to non-uniform thickness," J. Appl. Phys., vol. 89, pp. 7257-7259, 2001.

[8] J. Jorzick, S. O. Demokritov, B. Hillebrands, M. Bailleul, C. Fermon, K. Y. Guslienko, A. N. Slavin, D. V. Berkov, and N. L. Gorn, "Spin wave wells in nonellipsiodal micrometer size magnetic elements," Phys. Rev. Lett., vol. 88, p. 047204, 2002.

[9] J. P. Park, P. Eames, D. M. Engebretson, J. Berezovsky, and P. A. Crowell, "Spatially resolved dynamics of localized spin-wave modes in ferromagnetic wires," Phys. Rev. Lett., vol. 89, p. 277201, 2002.

[10] R. D. McMichael and M. D. Stiles, "Magnetic normal modes of nanoelements," J. Appl. Phys, vol. 97, p. 10J901, 2005. 